\title{
The global burden of nasopharyngeal carcinoma from 2009 to 2019: an observational study based on the Global Burden of Disease Study 2019
}

\author{
Hao $\mathrm{Yu}^{1} \cdot$ Xin Yin ${ }^{1} \cdot$ Yiran $\mathrm{Mao}^{2} \cdot$ Meiqin $\mathrm{Chen}^{3} \cdot$ Qiuying Tang ${ }^{1} \cdot$ Senxiang Yan ${ }^{1} \mathbb{D}$
}

Received: 21 March 2021 / Accepted: 29 May 2021 / Published online: 19 June 2021

(c) The Author(s) 2021

\begin{abstract}
Purpose The incidence and mortality rate of nasopharyngeal carcinoma (NPC) has changed in recent years. Our goal is to determine the epidemiological pattern of NPC to help policymakers allocate limited medical resources.

Methods Detailed information about NPC from 2009 to 2019 was collected from the Global Burden of Disease 2019 database. Age-standardized rates (ASRs) and corresponding estimated annual percentage changes (EAPCs) were calculated to assess NPC's incidence and mortality trends.

Results Globally, there was a consistent increase in the NPC incidence cases from 2009 to 2019 (from $121.65 \times 10^{3}$ cases in 2009 to $176.50 \times 10^{3}$ cases in 2019 , increasing by $45.09 \%$ ). The age-standardized incidence rate (ASIR) of NPC increased from 1.81 in 2009 to 2.12 in 2019 (EAPC $=1.59,95 \%$ CI 1.36-1.81). On the contrary, the mortality of NPC showed a downward trend (ASDR: 0.93 in 2009 and 0.86 in 2019; EAPC $=-0.63,95 \% \mathrm{CI}-0.78$ to -0.48 ), and it was negatively correlated with the social demographic index (SDI) in most regions. Both incidence and mortality rates of high-incidence territories tended to be stable or decline. Males had significantly higher incidence and mortality of NPC than females. The number of patients with onset age greater than 50 years old accounted for the highest proportion. We found that smoking, occupational exposure to formaldehyde, and alcohol use were the main risk factors for NPC-related mortality.

Conclusion Globally, the incidence rate of NPC has been slightly increasing, while the mortality and disability-adjusted life years (DALYs) have been decreasing. NPC burden in high-middle and middle SDI areas was the heaviest. The current prevention strategy should be repositioned, and some countries should formulate more targeted approaches to reduce the current burden of NPC.
\end{abstract}

Keywords Nasopharyngeal carcinoma (NPC) · Global Burden of Disease (GBD) · Incidence · Mortality · Age-standardized rate (ASR) - Estimated annual percentage change (EAPC)

\section{Introduction}

Hao Yu and Xin Yin contributed equally to this work.

Senxiang Yan

yansenxiang@zju.edu.cn

1 Department of Radiation Oncology, The First Affiliated Hospital, Zhejiang University School of Medicine, \#79 Qingchun Road, Hangzhou 310003, Zhejiang, People's Republic of China

2 School of Life Sciences, Tsinghua University, 30 Shuangqing Road, Haidian District, Beijing 100084, People's Republic of China

3 Department of Radiation Oncology, Affiliated Jinhua Hospital, College of Medicine, Zhejiang University, Jinhua 321000, Zhejiang, People's Republic of China
Nasopharyngeal carcinoma (NPC) originates from the nasopharyngeal epithelium and is divided into three major pathological subtypes: keratinizing squamous, non-keratinizing squamous and basaloid squamous cell carcinoma. Among them, non-keratinizing NPC can be divided into differentiated and undifferentiated tumors [1]. In the high incidence area of NPC, non-keratinizing carcinoma accounts for the majority, while in the low incidence area, keratinizing carcinoma is the primary type $[2,3]$. Compared with other cancers, NPC has a unique distribution pattern. In 2018, 129,000 new cases and 73,000 deaths of NPC were reported worldwide [4]. The geographic distribution of these cases was extremely unbalanced, with more than $70 \%$ of new cases 
occurring in East and Southeast Asia, while in most other regions, the age-standardized incidence rate of NPC in both males and females was less than $1 / 100,000$ person-years [1]. The incidence rate of NPC in males is $2-3$ times higher than females in almost all the populations surveyed [5].

The etiology of NPC is complex, including Epstein-Barr virus (EBV) infection, host genetics, and environmental factors [5]. EBV is ubiquitous in NPC cells, most closely related to the undifferentiated histological type of NPC, which is most common in southern China and Southeast Asia [6, 7]. However, the carcinogenic mechanism of EBV remains unclear, possibly due to the abnormal interaction between latent viral infection in epithelial cells and existing precancerous genetic changes [8]. In addition to viral infection, host genetic factors are also crucial causal agents for NPC. The human leukocyte antigen gene (HLA) located in the MHC region on chromosome 6p21 has been widely recognized as a major risk site for NPC [9]. The main environmental factors for NPC include smoking [10], alcohol consumption [11], eating salted fish and salted products [12, 13], while eating fresh fruits and vegetables is a protective factor for NPC [14].

To accommodate the rapid change in the epidemiological characteristics of NPC, a complete study to examine the epidemiological patterns of NPC at multiple levels is required, to comprehensively assess the distribution and development trend of NPC in different countries. In this study, we detailed NPC statistics of 204 countries or territories worldwide from 2009 to 2019, aiming to help policymakers assess the burden of disease and allocate the limited resources of the medical system accordingly.

\section{Materials and methods}

\section{Data sources}

The Global Burden of Disease (GBD) database contains statistics on 369 diseases in 204 countries or territories [15]. The data including incidence numbers, deaths, disabilityadjusted life years (DALYs), and corresponding age-standardized rates (ASRs) were downloaded from the Global Health Data Exchange (GHDx) query tool (http://ghdx. healthdata.org/gbd-results-tool). Information on gender, age, and potential risk factors associated with NPC was also downloaded to assess their impact on disease burden. Social demographic index (SDI) was used to divide countries or territories into five categories (high SDI, high-middle SDI, middle SDI, low-middle SDI and low SDI region). SDI values range from 0 to 1 , which indicates a country's degree of development based on the level of per capita income, education and total fertility rate [15].

\section{Statistical analysis}

Annual incidence cases, deaths, DALYs, ASRs and corresponding estimated annual percentage changes (EAPCs) were used to assess trends in the incidence and mortality rate of NPC. DALYs are composed of years of life lost (YLLs) and years lived with disability (YLDs), and the corresponding calculation formula is DALYs $=$ YLLs + YLDs. One DALY can be regarded as 1 lost year of "healthy life" [15]. ASRs include age-standardized incidence rate (ASIR), age-standardized death rate (ASDR) and age-standardized DALY rate. The ASR provided in GHDx is considered an objective indicator to quantify trends in cancer incidence. Standardization is required for comparison among several differently agestructured populations or for a certain population over time with its time-dependent age profiles. The trend of ASR can be reflected by EAPC value, which is often used to measure trends in disease and mortality rates, using a linear model on the log of the ASR [16]. According to the following regression model: $y=\beta_{0}+\beta_{1} x+\varepsilon$, where $y$ represents $\ln (\mathrm{ASR})$ and $x$ refers to the calendar year, $\mathrm{EAPC}=100 \times\left(\exp \left(\beta_{1}\right)-1\right)$ and its $95 \%$ confidence interval (CI) can be obtained. If the EAPC value and its lower limit of $95 \%$ CI are both positive, ASR is considered rising. Conversely, if the EAPC value and its upper limit of $95 \%$ CI are both negative, ASR shows a downward trend. Otherwise, ASR is considered stable. Finally, to examine the correlation between the trend of ASRs and the degree of social development, we calculated the Pearson correlation coefficient between EAPCs and SDI values.

\section{Data visualization}

All data analysis was based on open-source software R (version 3.6.3). Data cleaning was conducted using packages including dplyr and tidyr. Data visualization was performed using packages including ggplot2, Rcolor Brewer, and cowplot. Stacked histograms were used to show the trends of NPC incidence, death and DALY from 2009 to 2019. The world map was used to display the NPC burden of 204 countries or territories visually. Bubble plots and scatter plots were used and regression curves were added to analyze the correlation between ASRs and SDI values as well as that between EAPCs and SDI values. Histograms were used to show the differences in incidence and mortality between males and females in territories with high incidence of NPC. The dynamic distribution of age composition and mortalityrelated risk factors in NPC patients was represented by area graphs. 


\section{Results}

\section{Change in the incidence of NPC}

There was a consistent yearly increase in the NPC incidence from 2009 to 2019 worldwide (from $121.65 \times 10^{3}$ cases in 2009 to $176.50 \times 10^{3}$ cases in 2019 , increasing by $45.09 \%$ ) (Fig. 1A) (Table 1). There were more male patients than female ones (male to female ratio $=2.27: 1$ in 2009, and 2.59:1 in 2019). The global incidence rate of NPC also showed an increasing trend (ASIR: 1.81 in 2009 and 2.12 in 2019 , EAPC $=1.59,95 \%$ CI $1.36-1.81$ ). Subgroup analysis by SDI demonstrated that high-middle SDI area had the highest burden of NPC (incidence cases: $46.12 \times 10^{3}$ in 2009 and $68.02 \times 10^{3}$ in 2019; ASIR: 2.86 in 2009 and 3.58 in 2019 , EAPC $=2.46,95 \%$ CI $2.13-2.79$ ). ASIR decreased the fastest in the low SDI areas (ASIR: 0.88 in 2009 and 0.85 in 2019, $\mathrm{EAPC}=-0.28,95 \% \mathrm{CI}$ -0.47 to -0.08 ), and plateaued in high SDI areas (ASIR: 1.29 in 2009 and 1.29 in 2019, $\mathrm{EAPC}=0.00$, 95\% CI -0.17 to 0.17 ). At the intercontinental level, East Asia and South Asia had the most incidence cases (East Asia: $71.34 \times 10^{3}$ cases in 2009 and $113.40 \times 10^{3}$ cases in 2019 ; South Asia: $11.92 \times 10^{3}$ cases in 2009 and $15.78 \times 10^{3}$ cases in 2019). ASIR had increased in East Asia, Central Asia, the Middle East, and Eastern Europe, and the two fastest-growing areas were East Asia and Eastern Europe. ASIR tended to be stable or decreased in most other regions, especially in southern Sub-Sahara Africa and Tropical Latin America (EAPC $=-1.58$ and -1.09 , respectively) (Fig. 3A). At the national or regional level, the top three countries of incidence cases were China, India and Japan, all located in Asia $\left(68.78 \times 10^{3}\right.$, $9.13 \times 10^{3}$, and $3.74 \times 10^{3}$ cases in 2009 , respectively; $110.43 \times 10^{3}, 12.21 \times 10^{3}, 3.98 \times 10^{3}$ cases in 2019 , respectively) (Fig. 2A) (Tables S1, S7). Singapore and Taiwan (province of China) had the highest ASIR both in 2009 and 2019 (Singapore: ASIR = 12.64 in 2009 and 10.81 in 2019; Taiwan: ASIR = 6.77 in 2009 and 7.14 in 2019) (Tables S4, S10, Fig. S1A). Ukraine had the most significant increase in ASIR (EAPC $=3.68,95 \%$ CI 2.49-4.87) (Table S13).

\section{Change in NPC mortality}

Globally, although the NPC-related deaths were increasing yearly, the ASDR was declining (ASDR: 0.93 in 2009 and 0.86 in 2019; $\mathrm{EAPC}=-0.63,95 \% \mathrm{CI}-0.78$ to -0.48 ) (Fig. 1B) (Table 2). The number of deaths in males was higher than that in females (male to female ratio $=2.46: 1$ in 2009, and 2.51:1 in 2019). Subgroup analysis by SDI demonstrated that the middle SDI region had the most NPC-related deaths $\left(24.62 \times 10^{3}\right.$ cases in 2009 and $28.75 \times 10^{3}$ cases in 2019). ASDR decreased in all five SDI regions, especially in middle SDI and high SDI areas $(\mathrm{EAPC}=-1.28$ and -0.54 , respectively $)$. At the intercontinental level, three regions with the highest number of NPC-related deaths were all located in Asia (deaths in 2019: East Asia: $30.10 \times 10^{3}$; South Asia: $14.66 \times 10^{3}$; Southeast Asia: $11.26 \times 10^{3}$ ). ASDR in most areas showed a downward trend, among which Tropical Latin America and southern Sub-Sahara Africa showed the fastest decline (Tropical Latin America: EAPC $=-1.74,95 \%$ CI -1.92 to -1.56 ; Southern Sub-Saharan Africa: $\mathrm{EAPC}=-1.70$, $95 \% \mathrm{CI}-2.00$ to -1.40$)$. At the national or regional level, China, India and Indonesia had the largest number of deaths $\left(26.03 \times 10^{3}, 8.60 \times 10^{3}\right.$, and $2.69 \times 10^{3}$ cases in 2009 , respectively; $28.66 \times 10^{3}, 11.36 \times 10^{3}, 3.22 \times 10^{3}$ cases in 2019, respectively) (Fig. 2B) (Tables S2, S8). Greenland and Malaysia were two regions with the highest ASDR both in 2009 and 2019 (Greenland: ASDR $=5.69$ in 2009 and 4.68 in 2019; Malaysia: ASDR $=4.21$ in 2009 and 4.76 in 2019) (Tables S5, S11, Fig. S1B). Although the EAPC values of a small part of regions were positive, the lower limits of $95 \%$ CI of EAPC values of all countries or territories were negative. Therefore, ASDR of all countries and territories in the world tended to be stable or declining. ASDR of high incidence territories of NPC such as East Asia, Southeast Asia, South Asia, North Africa and the Middle East also decreased to varying degrees (Fig. 3B) (Table S14).

\section{Change in DALYs for NPC}

Globally, the DALYs were increasing from $2046.98 \times 10^{3}$ in 2009 to $2335.10 \times 10^{3}$ in 2019 , while the age-standardized DALY rate declined (30.22 in 2009 and 27.98 in 2019, $\mathrm{EAPC}=-0.60,95 \% \mathrm{CI}-0.79$ to -0.42$)$ (Fig. $1 \mathrm{C}$, Table 3). Males were still the main component (male to female ratio $=2.50: 1$ in 2009 and 2.59:1 in 2019). Subgroup analysis by SDI demonstrated that the middle SDI region had the highest DALY $\left(814.13 \times 10^{3}\right.$ in 2009 and $914.42 \times 10^{3}$ in 2019). The age-standardized DALY rate decreased in all regions, especially in middle SDI and high SDI areas (EAPC $=-1.21$ and -0.56 , respectively). At the intercontinental level, DALYs were all gradually increasing. East Asia had the highest DALY $\left(902.48 \times 10^{3}\right.$ in 2009 and $958.33 \times 10^{3}$ in 2019). However, the agestandardized DALY rate decreased in most regions including East Asia, especially in southern Sub-Saharan Africa $(\mathrm{EAPC}=-1.83,95 \% \mathrm{CI}-2.03$ to -1.63$)$. At the national or regional level, China, India and Indonesia had the most DALY $\left(858.85 \times 10^{3}, 306.54 \times 10^{3}\right.$, and $88.62 \times 10^{3}$ 
A
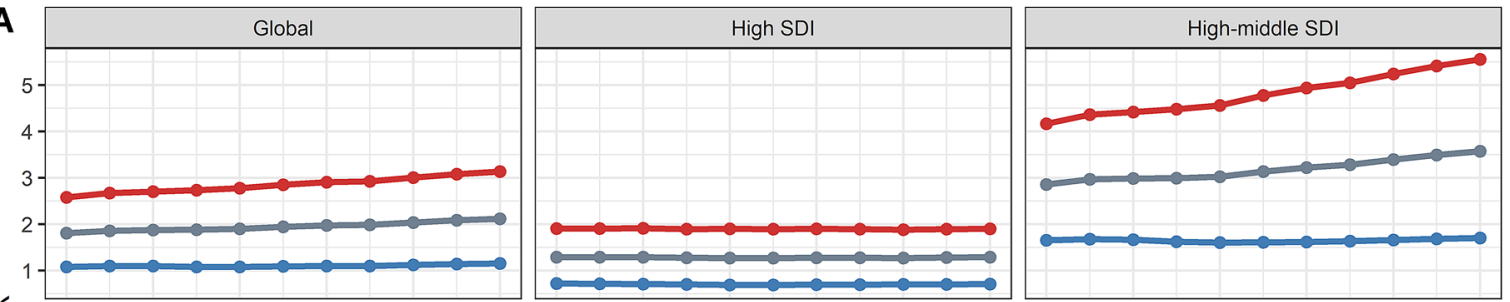

$\frac{\underline{c}}{\text { 是 }}$
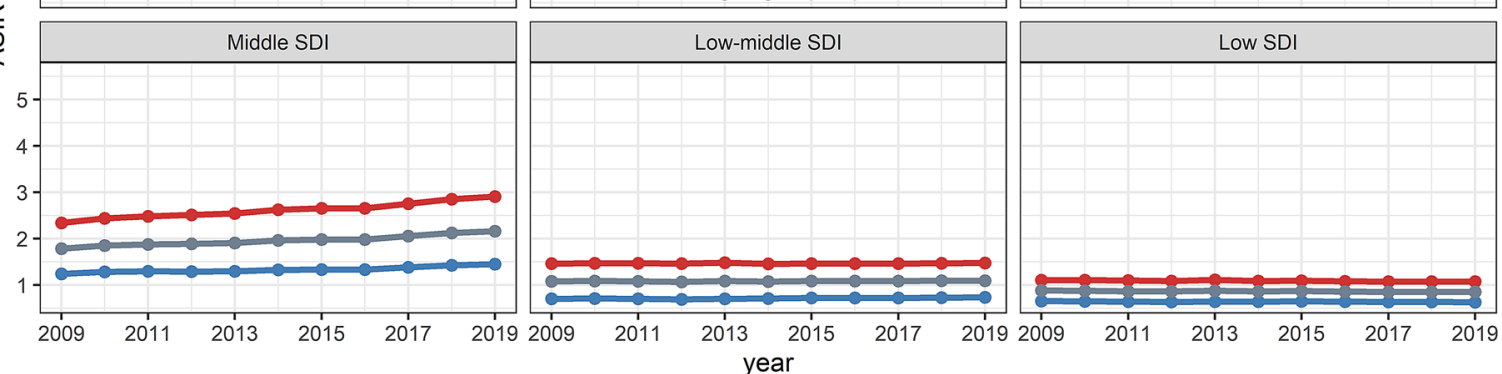

Sex

B

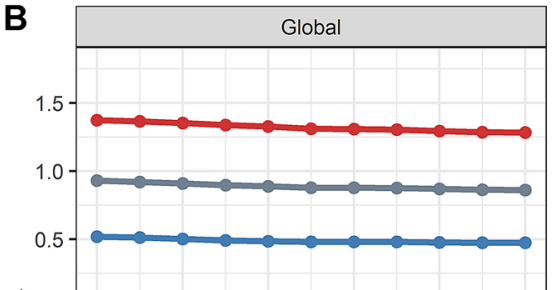

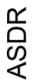

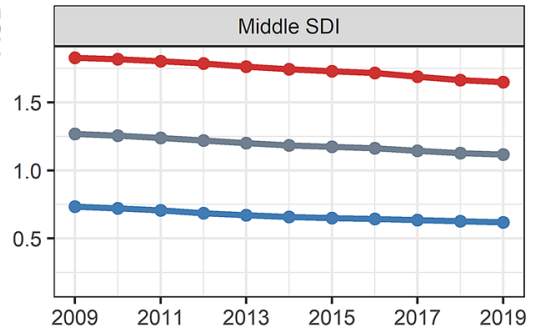

C
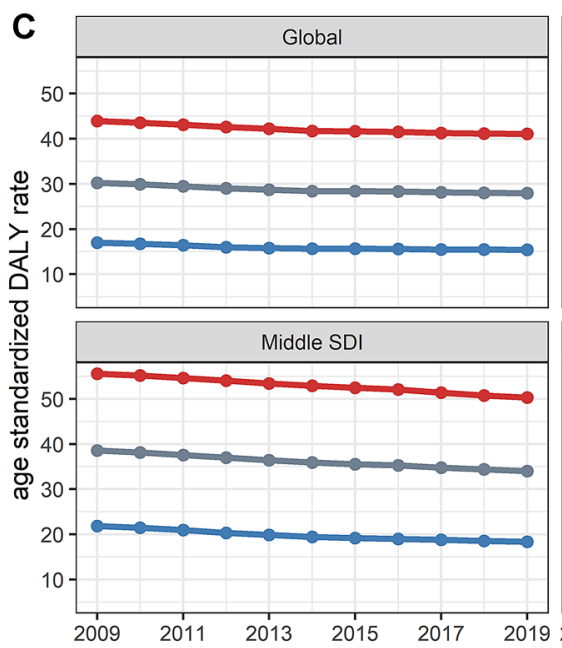

$\begin{array}{llllll}2009 & 2011 & 2013 & 2015 & 2017 & 20192009\end{array}$
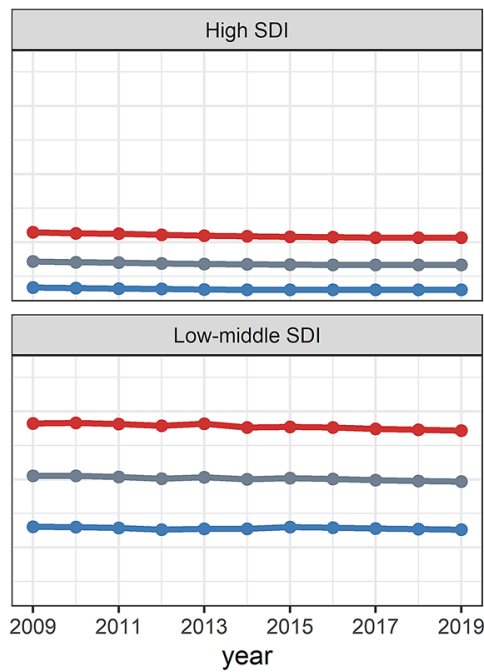

year
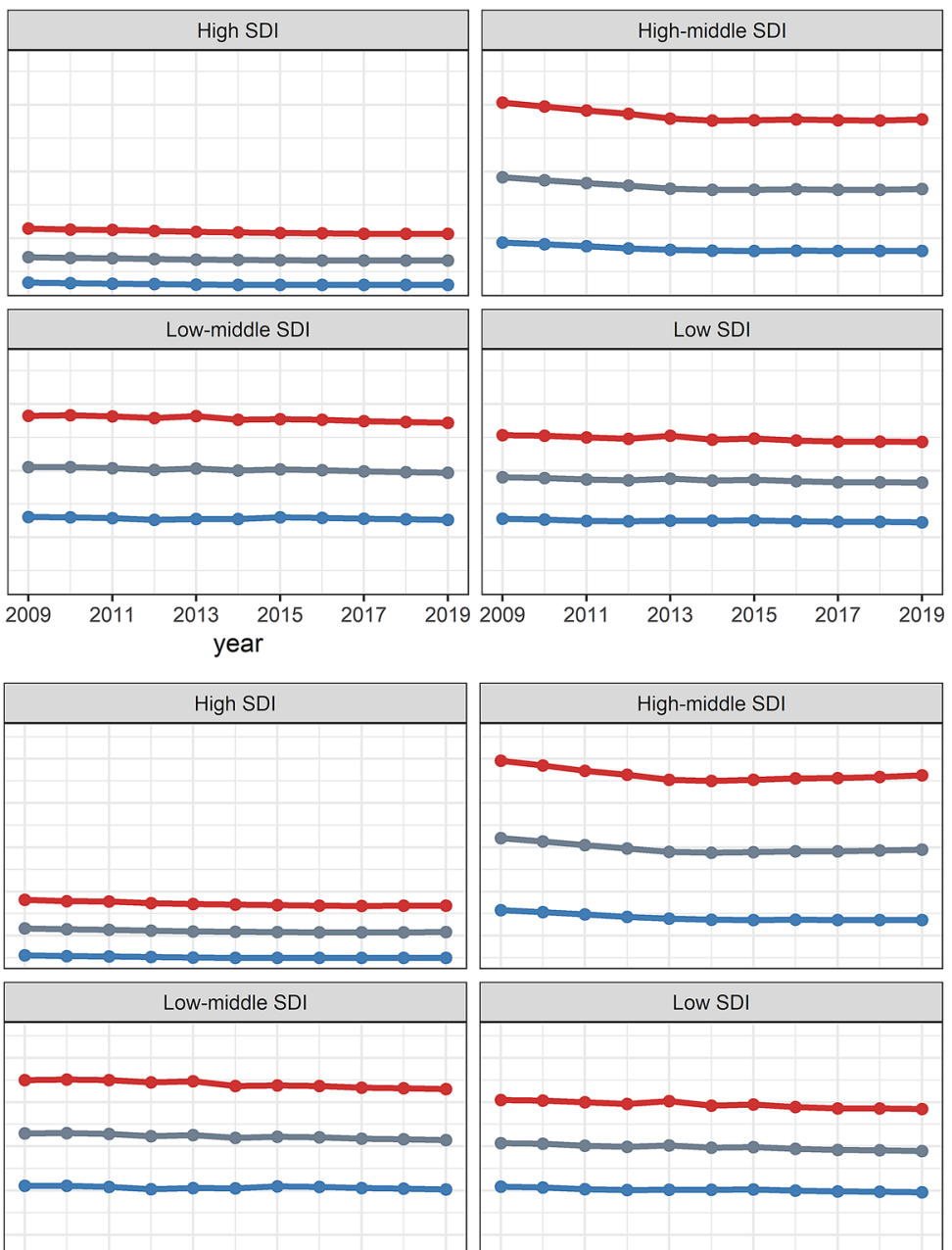

Sex

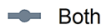

- Male

$\stackrel{\sim}{=}$ Female
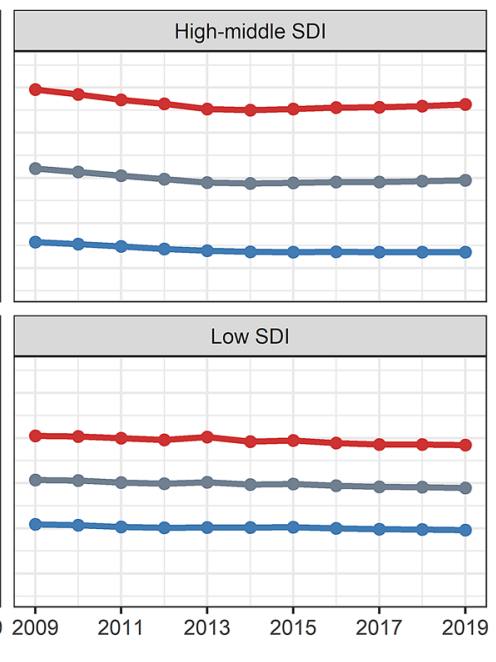

Sex

$\leadsto$ Both

$\rightarrow$ Male

- Female

Fig. 1 The change trends of NPC's ASIR, ASDR, and age-standardized DALY rate from 2009 to 2019. A The change trends of ASIR. B The change trends of ASDR. $\mathbf{C}$ The change trends of age-standardized DALY rate 
Table 1 The incident cases and ASIR of NPC in 2009/2019 and its temporal trends

\begin{tabular}{|c|c|c|c|c|c|}
\hline & \multicolumn{2}{|l|}{2009} & \multicolumn{2}{|l|}{2019} & \multirow{2}{*}{$\begin{array}{l}\text { 2009-2019 } \\
\text { EAPC } \\
\text { No. }(95 \% \text { CI })\end{array}$} \\
\hline & $\begin{array}{l}\text { Incidence cases } \\
\text { No } * 10^{3}(95 \% \mathrm{CI})\end{array}$ & $\begin{array}{l}\text { ASIR/100,000 } \\
\text { No. }(95 \% \mathrm{CI})\end{array}$ & $\begin{array}{l}\text { Incidence cases } \\
\text { No } * 10^{3}(95 \% \mathrm{CI})\end{array}$ & $\begin{array}{l}\text { ASIR/100,000 } \\
\text { No. }(95 \% \text { CI })\end{array}$ & \\
\hline Global & $121.65(114.34-130.12)$ & $1.81(1.70-1.93)$ & $176.50(156.05-199.92)$ & $2.12(1.87-2.40)$ & $1.59(1.36-1.81)$ \\
\hline Male & 84.48 (78.14-91.91) & $2.58(2.40-2.80)$ & $127.28(108.04-148.52)$ & $3.14(2.67-3.65)$ & $1.90(1.73-2.08)$ \\
\hline Female & $37.17(34.28-40.10)$ & $1.08(1.00-1.16)$ & $49.22(42.60-56.98)$ & $1.16(1.00-1.34)$ & $0.78(0.40-1.17)$ \\
\hline High SDI & $16.76(16.15-17.33)$ & $1.29(1.25-1.34)$ & $19.27(17.28-21.48)$ & $1.29(1.16-1.44)$ & $0.00(-0.17$ to 0.17$)$ \\
\hline High-middle SDI & $46.12(41.54-51.30)$ & $2.86(2.58-3.17)$ & $68.02(56.47-81.57)$ & $3.58(2.98-4.28)$ & $2.46(2.13-2.79)$ \\
\hline Middle SDI & $37.03(34.41-40.34)$ & $1.78(1.66-1.93)$ & $57.53(50.14-65.91)$ & $2.16(1.89-2.47)$ & $1.80(1.41-2.18)$ \\
\hline Low-middle SDI & $12.43(11.43-13.53)$ & $1.08(0.99-1.17)$ & $16.59(15.07-18.48)$ & $1.09(1.00-1.22)$ & $0.21(0.07-0.35)$ \\
\hline Low SDI & $4.10(3.64-4.58)$ & $0.88(0.78-0.98)$ & $5.38(4.77-5.97)$ & $0.85(0.75-0.94)$ & $-0.28(-0.47$ to -0.08$)$ \\
\hline Andean Latin America & $0.08(0.07-0.09)$ & $0.18(0.16-0.20)$ & $0.10(0.08-0.13)$ & $0.18(0.14-0.22)$ & $-0.55(-0.76$ to -0.35$)$ \\
\hline Australasia & $0.28(0.26-0.30)$ & $0.83(0.78-0.90)$ & $0.30(0.24-0.38)$ & $0.75(0.60-0.93)$ & $-0.84(-1.27$ to -0.41$)$ \\
\hline Caribbean & $0.26(0.24-0.29)$ & $0.62(0.56-0.68)$ & $0.33(0.28-0.39)$ & $0.65(0.55-0.75)$ & $0.62(0.42-0.82)$ \\
\hline Central Asia & $0.29(0.28-0.30)$ & $0.42(0.41-0.44)$ & $0.39(0.35-0.45)$ & $0.46(0.41-0.52)$ & $0.99(0.43-1.55)$ \\
\hline Central Europe & $1.04(1.00-1.08)$ & $0.64(0.62-0.66)$ & $1.03(0.90-1.19)$ & $0.62(0.54-0.72)$ & $-0.29(-0.53$ to -0.04$)$ \\
\hline Central Latin America & $0.43(0.41-0.44)$ & $0.23(0.22-0.24)$ & $0.56(0.48-0.67)$ & $0.23(0.20-0.27)$ & $-0.01(-0.41$ to 0.40$)$ \\
\hline $\begin{array}{l}\text { Central Sub-Saharan } \\
\text { Africa }\end{array}$ & $0.24(0.19-0.29)$ & $0.52(0.42-0.64)$ & $0.33(0.25-0.42)$ & $0.51(0.39-0.65)$ & $-0.40(-0.51$ to -0.30$)$ \\
\hline East Asia & $71.34(64.54-79.06)$ & $4.21(3.82-4.64)$ & $113.40(93.19-135.38)$ & $5.61(4.66-6.68)$ & $2.74(2.44-3.04)$ \\
\hline Eastern Europe & $1.35(1.30-1.41)$ & $0.47(0.46-0.49)$ & $1.51(1.33-1.70)$ & $0.53(0.47-0.59)$ & $1.84(0.82-2.86)$ \\
\hline $\begin{array}{l}\text { Eastern Sub-Saharan } \\
\text { Africa }\end{array}$ & $1.89(1.49-2.21)$ & $1.25(0.98-1.47)$ & $2.54(1.91-3.11)$ & $1.21(0.90-1.47)$ & $-0.36(-0.44$ to -0.28$)$ \\
\hline High-income Asia Pacific & $5.29(4.95-5.59)$ & $1.80(1.70-1.89)$ & $5.98(5.14-6.93)$ & $1.73(1.50-1.98)$ & $-0.63(-0.84$ to -0.42$)$ \\
\hline $\begin{array}{l}\text { High-income North } \\
\text { America }\end{array}$ & $3.13(3.01-3.24)$ & $0.71(0.68-0.73)$ & $3.53(3.03-4.10)$ & $0.69(0.60-0.80)$ & $-0.13(-0.26$ to 0.00$)$ \\
\hline $\begin{array}{l}\text { North Africa and Middle } \\
\text { East }\end{array}$ & $4.44(3.94-4.99)$ & $1.14(1.01-1.27)$ & $6.64(5.78-7.69)$ & $1.25(1.09-1.44)$ & $0.90(0.79-1.00)$ \\
\hline Oceania & $0.08(0.06-0.10)$ & $1.26(0.95-1.59)$ & $0.11(0.08-0.14)$ & $1.28(0.95-1.64)$ & $0.04(-0.11$ to 0.20$)$ \\
\hline South Asia & $11.92(10.84-13.13)$ & $1.02(0.93-1.12)$ & $15.78(13.77-18.20)$ & $1.01(0.88-1.16)$ & $-0.10(-0.20$ to 0.00$)$ \\
\hline Southeast Asia & 9.85 (9.04-10.73) & $1.93(1.77-2.09)$ & $13.12(11.40-15.08)$ & $1.95(1.70-2.23)$ & $0.16(-0.05$ to 0.36$)$ \\
\hline Southern Latin America & $0.18(0.17-0.20)$ & $0.28(0.26-0.30)$ & $0.23(0.18-0.29)$ & $0.29(0.23-0.37)$ & $0.41(-0.10$ to 0.93$)$ \\
\hline $\begin{array}{l}\text { Southern Sub-Saharan } \\
\text { Africa }\end{array}$ & $0.28(0.26-0.31)$ & $0.59(0.55-0.64)$ & $0.31(0.27-0.34)$ & $0.50(0.45-0.55)$ & $-1.58(-1.85$ to -1.31$)$ \\
\hline Tropical Latin America & $0.59(0.57-0.61)$ & $0.30(0.29-0.31)$ & $0.69(0.64-0.73)$ & $0.28(0.26-0.30)$ & $-1.09(-1.30$ to -0.87$)$ \\
\hline Western Europe & $7.67(7.32-8.02)$ & $1.26(1.20-1.32)$ & $8.24(7.01-9.63)$ & $1.21(1.03-1.43)$ & $-0.28(-0.52$ to -0.05$)$ \\
\hline $\begin{array}{l}\text { Western Sub-Saharan } \\
\text { Africa }\end{array}$ & $1.01(0.80-1.29)$ & $0.59(0.47-0.74)$ & $1.38(1.09-1.71)$ & $0.57(0.46-0.71)$ & $-0.28(-0.67$ to 0.11$)$ \\
\hline
\end{tabular}

ASIR age-standardized incidence rate

in 2009 , respectively; $912.11 \times 10^{3}, 388.09 \times 10^{3}$, $100.80 \times 10^{3}$ in 2019 , respectively) (Fig. 2C) (Tables S3, S9). Malaysia and Greenland were two countries with the highest age-standardized DALY rates both in 2009 and 2019 (Greenland: 171.99 in 2009 and 137.74 in 2019; Malaysia: 131.31 in 2009 and 152.29 in 2019) (Tables S6, S12, Fig. S1C). Just like ASDR, the Age-standardized DALY rate plateaued or decreased in all countries or territories worldwide (Fig. 3C) (Table S15).

\section{The correlation between SDI and global NPC burden}

We explored the correlation between SDI in 2019 and EAPCs of ASRs in 204 countries or territories. The results demonstrated that negative correlation occurred between SDI and EAPC values of all ASRs, and the correlation between SDI and EAPCs of ASDR/age-standardized DALY rate was statistically significant (correlation coefficient $=-0.35$ and -0.33 , respectively. All $P$ 


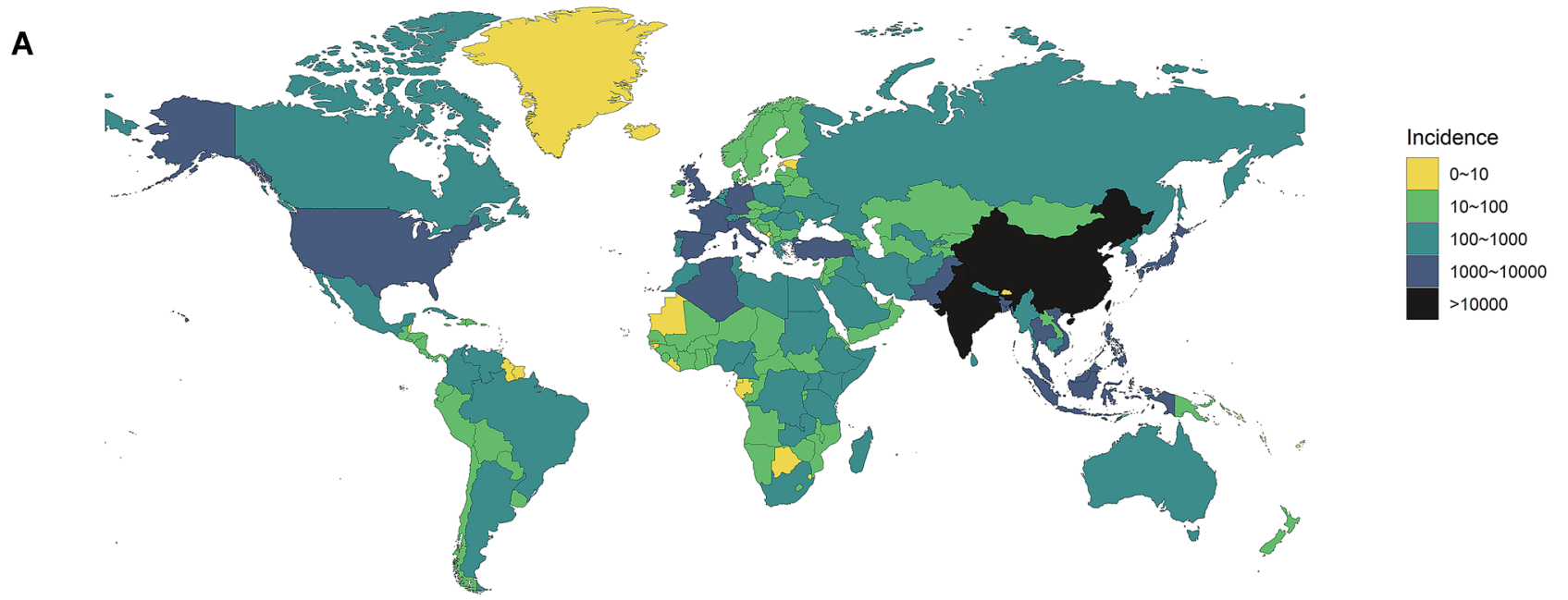

B

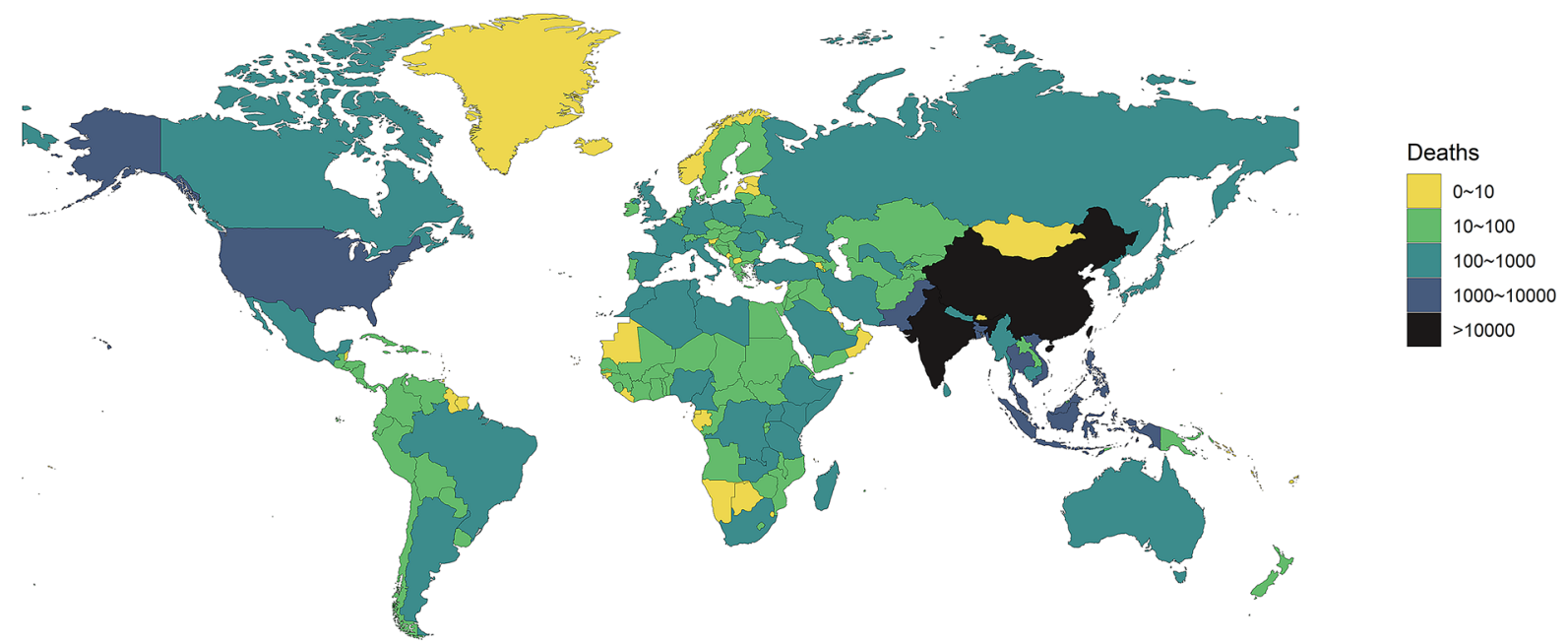

C

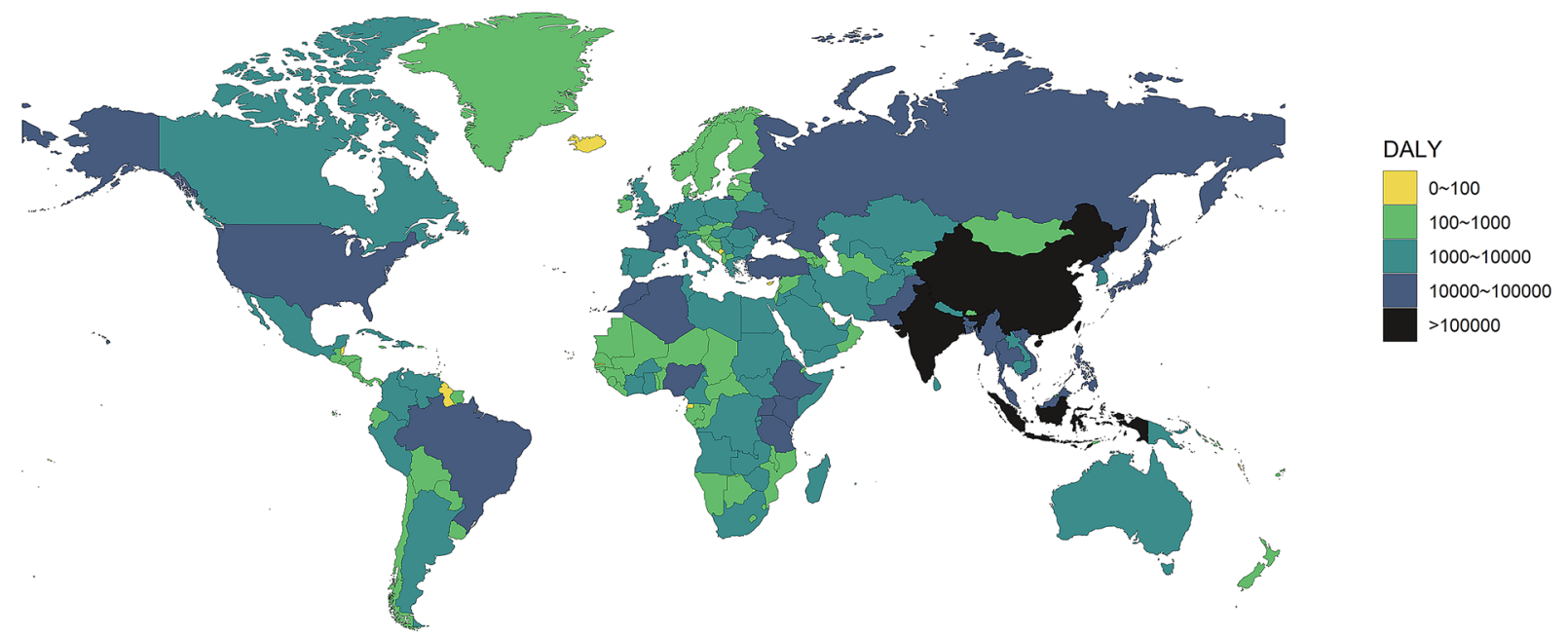

Fig. 2 The global disease burden of NPC for both sexes in 204 countries or territories. A The incidence cases of NPC in 2019. B The deaths of NPC in 2019. C The DALYs of NPC in 2019 
Table 2 The deaths and ASDR of NPC in 2009/2019 and its temporal trends

\begin{tabular}{|c|c|c|c|c|c|}
\hline & \multicolumn{2}{|l|}{2009} & \multicolumn{2}{|l|}{2019} & \multirow{2}{*}{$\begin{array}{l}2009-2019 \\
\text { EAPC } \\
\text { No. }(95 \% \text { CI })\end{array}$} \\
\hline & $\begin{array}{l}\text { Deaths cases } \\
\text { No } * 10^{3}(95 \% \text { CI })\end{array}$ & $\begin{array}{l}\text { ASDR/100,000 } \\
\text { No. }(95 \% \text { CI })\end{array}$ & $\begin{array}{l}\text { Deaths cases } \\
\text { No } * 10^{3}(95 \% \mathrm{CI})\end{array}$ & $\begin{array}{l}\text { ASDR/100,000 } \\
\text { No. }(95 \% \text { CI })\end{array}$ & \\
\hline Slobal & $60.78(57.52-64.14)$ & $0.93(0.88-0.98)$ & $71.61(65.44-77.62)$ & $0.86(0.79-0.93)$ & $-0.63(-0.78$ to -0.48$)$ \\
\hline Male & $43.21(40.60-46.14)$ & $1.38(1.29-1.47)$ & $51.22(45.97-56.95)$ & $1.28(1.15-1.43)$ & $-0.64(-0.76$ to -0.53$)$ \\
\hline Female & $17.57(16.09-18.84)$ & $0.52(0.48-0.56)$ & $20.39(18.16-22.78)$ & $0.47(0.42-0.53)$ & $-0.61(-0.84$ to -0.37$)$ \\
\hline High SDI & $4.98(4.78-5.11)$ & $0.36(0.35-0.37)$ & $5.55(5.14-5.98)$ & $0.33(0.31-0.36)$ & $-0.54(-0.81$ to -0.27$)$ \\
\hline High-middle SDI & $15.42(14.34-16.71)$ & $0.96(0.89-1.04)$ & $17.22(14.92-19.68)$ & $0.87(0.75-0.99)$ & $-0.53(-0.98$ to -0.07$)$ \\
\hline Middle SDI & $24.62(23.10-26.53)$ & $1.27(1.19-1.36)$ & $28.75(25.63-32.05)$ & $1.12(1.00-1.24)$ & $-1.28(-1.35$ to -1.20$)$ \\
\hline Low-middle SDI & $11.47(10.54-12.45)$ & $1.03(0.94-1.11)$ & $14.51(13.15-16.11)$ & $0.98(0.89-1.09)$ & $-0.37(-0.54$ to -0.19$)$ \\
\hline Low SDI & $4.25(3.82-4.73)$ & $0.95(0.86-1.06)$ & $5.54(4.90-6.23)$ & $0.91(0.81-1.02)$ & $-0.35(-0.53$ to -0.17$)$ \\
\hline Andean Latin America & $0.07(0.06-0.08)$ & $6-0.19)$ & $0.11)$ & $-0.19)$ & $-1.20(-1.35$ to -1.04$)$ \\
\hline Australasia & $0.12(0.11-0.12)$ & $0.33(0.31-0.35)$ & $0.13(0.12-0.15)$ & $0.30(0.27-0.33)$ & $-0.94(-1.18$ to -0.70$)$ \\
\hline Caribbean & ) & $-0.58)$ & $3-0.31)$ & $1-0.60)$ & $0.43)$ \\
\hline Central Asia & $0.26(0.25-0.27)$ & $38-0.41)$ & $0.34(0.30-0.38)$ & 0.41 & 1.13) \\
\hline Central Europe & $0.80(0.77-0.82)$ & $5-0.48)$ & $0.74(C$ & $35-0.45)$ & $-1.52(-1.77$ to -1.28$)$ \\
\hline Central Latin America & 37) & $-0.21)$ & 0.4 & $-0.22)$ & $-0.79(-1.24$ to -0.35$)$ \\
\hline Central Su & $(0.19-0.28)$ & $-0.65)$ & $2(0.24-0.40)$ & $-0.66)$ & $-0.43(-0$ \\
\hline East Asia & $27.34(25.11-29.88)$ & $1.71(1.57-1.86)$ & $30.10(25.23-35.36)$ & $1.45(1.22-1.70)$ & $-1.68(-1.92$ to -1.44$)$ \\
\hline Eastern Europe & $0.95(0.93-0.97)$ & $0.32(0.31-0.33)$ & $0.96(0.84-1.09)$ & $0.31(0.27-0.35)$ & $0.07(-0.66-0.81)$ \\
\hline Eastern Sub-Saharan Africa & $1.80(1.41-2.10)$ & $1.25(0.97-1.46)$ & $2.41(1.81-2.94)$ & $1.20(0.91-1.46)$ & $-0.40(-0.45$ to -0.35$)$ \\
\hline High-income Asia Pacific & $1.16(1.08-1.21)$ & $0.36(0.34-0.37)$ & $1.30(1.17-1.41)$ & $0.32(0.29-0.35)$ & $-1.31(-1.52$ to -1.10$)$ \\
\hline High-income North America & $1.09(1.05-1.12)$ & $0.23(0.23-0.24)$ & $1.27(1.21-1.32)$ & $0.22(0.21-0.23)$ & $-0.34(-0.54$ to -0.14$)$ \\
\hline North Africa and Middle East & $2.40(2.11-2.72)$ & $0.69(0.61-0.78)$ & $2.95(2.57-3.37)$ & $0.61(0.54-0.70)$ & $-1.10(-1.18$ to -1.02$)$ \\
\hline Oceania & $0.07(0.05-0.09)$ & $1.23(0.93-1.55)$ & $0.10(0.08-0.13)$ & $1.25(0.94-1.60)$ & $0.06(-0.10-0.22)$ \\
\hline South Asia & $11.22(10.24-12.33)$ & $0.99(0.90-1.08)$ & $14.66(12.96-16.86)$ & $0.96(0.85-1.10)$ & $-0.28(-0.39$ to -0.17$)$ \\
\hline Southeast Asia & $8.90(8.18-9.70)$ & $1.83(1.68-1.98)$ & $11.26(9.87-12.85)$ & $1.74(1.53-1.98)$ & $-0.43(-0.58$ to -0.28$)$ \\
\hline Southern Latin America & $0.14(0.13-0.14)$ & $0.21(0.19-0.22)$ & $0.15(0.14-0.16)$ & $0.19(0.17-0.20)$ & $-0.97(-1.43$ to -0.51$)$ \\
\hline Southern Sub-Saharan Africa & $0.27(0.25-0.29)$ & $0.59(0.55-0.63)$ & $0.29(0.26-0.32)$ & $0.49(0.44-0.54)$ & $-1.70(-2.00$ to -1.40$)$ \\
\hline Tropical Latin America & $0.48(0.47-0.50)$ & $0.25(0.25-0.26)$ & $0.54(0.50-0.57)$ & $0.22(0.20-0.23)$ & $-1.74(-1.92$ to -1.56$)$ \\
\hline Western Europe & $1.95(1.88-2.01)$ & $0.29(0.28-0.30)$ & $1.99(1.87-2.11)$ & $0.26(0.24-0.27)$ & $-1.19(-1.42$ to -0.96$)$ \\
\hline Western Sub-Saharan Africa & $0.96(0.75-1.22)$ & $0.58(0.46-0.73)$ & $1.30(1.01-1.59)$ & $0.57(0.45-0.69)$ & $-0.33(-0.71$ to 0.05$)$ \\
\hline
\end{tabular}

$A S D R$ age-standardized death rate

values $<0.0001$ ) (Fig. 4A-C). In Fig. 4A, there was a turning point when SDI value was between 0.6 and 0.8 , which may be associated with the higher value of EAPC of ASIR in high-middle SDI region (EAPC $=2.46,95 \% \mathrm{CI}$ 2.13-2.79). We further explored the relationship between SDI in 2019 and NPC burden in 21 geographical regions from 2009 to 2019 (Fig. 4D-F). In general, there was a statistically significant negative linear relationship between SDI and ASDR/age-standardized DALY rate (correlation coefficient $=-0.46$ and -0.46 , respectively. All $P$ values $<0.0001)$. However, the relationship between SDI and ASIR was positive (correlation coefficient $=0.049$, $P$ value $>0.1)$. There was a peak in the curve when SDI value was between 0.6 and 0.7 , which may be related to the data of East Asia.

\section{NPC burden of high-incidence territories}

The ASIR is considered an objective indicator to quantify trends in cancer incidence. We selected the top 30 countries or territories with the highest ASIR of NPC in 2009, and defined them as "High-incidence territories of NPC". The results showed that while EAPCs of ASIRs were positive in 13 countries and territories, the lower limits of $95 \%$ CI of EAPCs in all high-incidence territories were negative, which indicated that although EAPC values were positive, ASIRs of these 13 countries or territories tended to be stable. ASIRs of the remaining 17 countries or territories showed a downward trend (Fig. 5A). Similarly, the lower limits of 95\% CI of EAPCs of ASDRs in all 30 territories were negative, indicating 
A

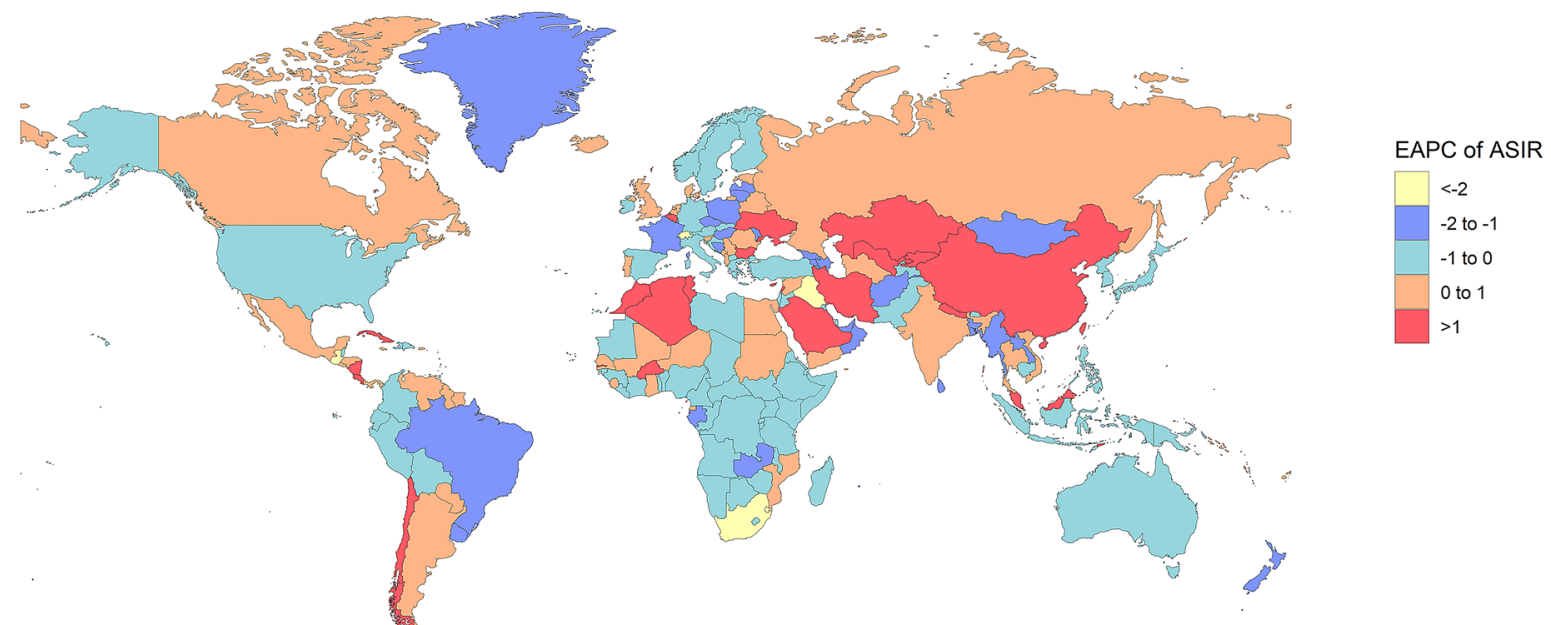

B
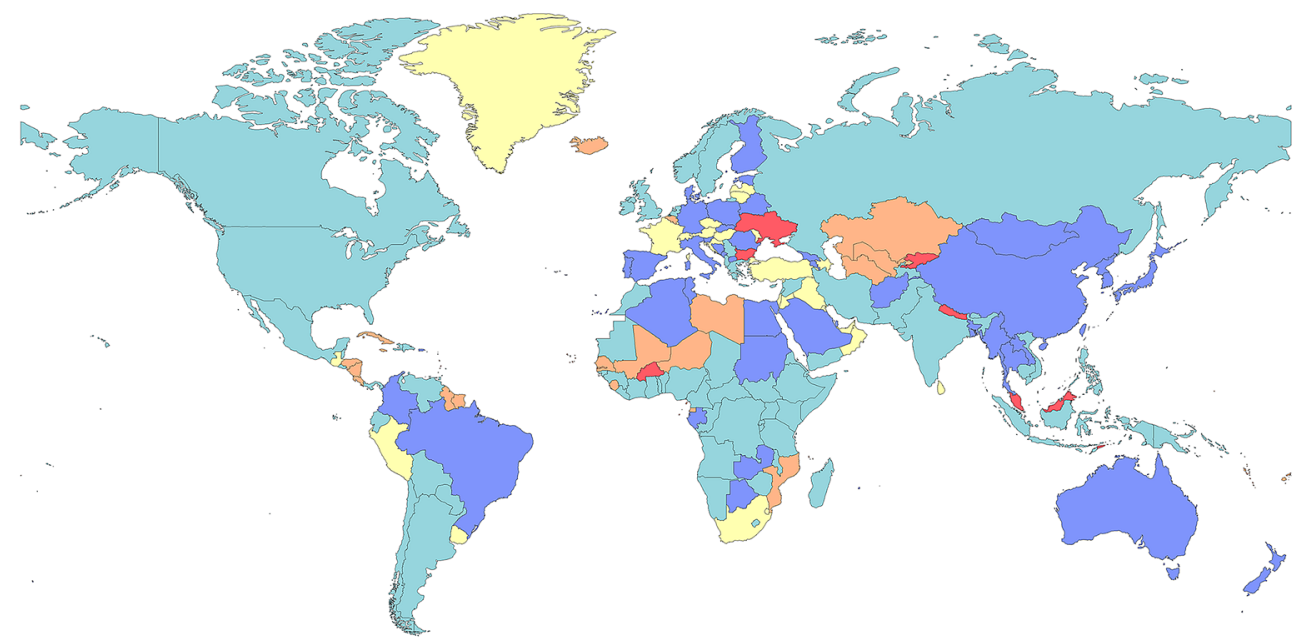

EAPC of ASDR

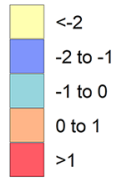

C
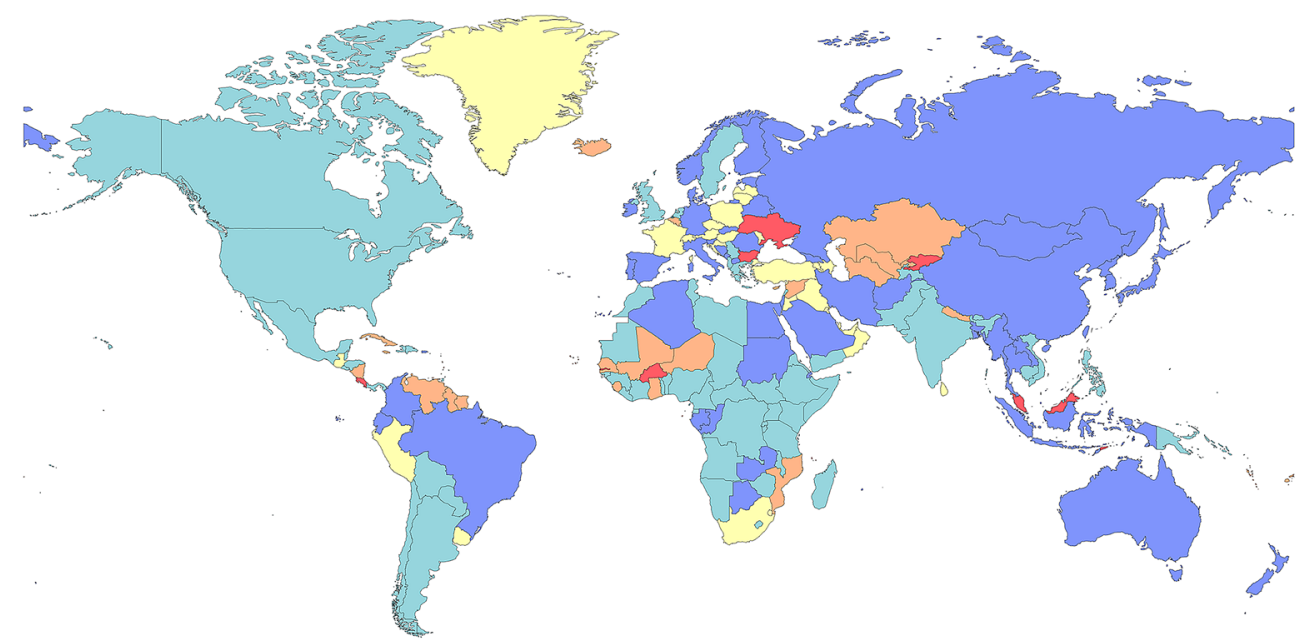

EAPC of age-standardized DALY rate

$\square<-2$

-2 to -1

-1 to 0

0 to 1

$>1$

Fig. 3 The EAPCs of NPC in 204 countries or territories. A The EAPC of ASIR from 2009 to 2019. B The EAPC of ASDR from 2009 to 2019. C The EAPC of age-standardized DALY rate from 2009 to 2019 
Table 3 The DALYs and age-standardized DALY rate of NPC in 2009/2019 and its temporal trends

\begin{tabular}{|c|c|c|c|c|c|}
\hline & \multicolumn{2}{|l|}{2009} & \multicolumn{2}{|l|}{2019} & \multirow{2}{*}{$\begin{array}{l}2009-2019 \\
\text { EAPC } \\
\text { No. }(95 \% \text { CI })\end{array}$} \\
\hline & $\begin{array}{l}\text { DALY } \\
\text { No } * 10^{3}(95 \% \text { CI })\end{array}$ & $\begin{array}{l}\text { Age-standardized } \\
\text { DALY rate/100,000 } \\
\text { No. }(95 \% \text { CI) }\end{array}$ & $\begin{array}{l}\text { DALY } \\
\text { No } * 10^{3}(95 \% \text { CI })\end{array}$ & $\begin{array}{l}\text { Age-standardized } \\
\text { DALY rate/100,000 } \\
\text { No. }(95 \% \text { CI })\end{array}$ & \\
\hline Global & $\begin{array}{l}2046.98(1935.69- \\
2172.48)\end{array}$ & $30.22(28.59-32.04)$ & $\begin{array}{l}2335.10(2139.75- \\
2536.66)\end{array}$ & $27.98(25.65-30.37)$ & $\begin{array}{l}-0.60(-0.79 \text { to } \\
-0.42)\end{array}$ \\
\hline Male & $\begin{array}{l}1461.53(1371.90- \\
1569.63)\end{array}$ & $43.94(41.29-47.08)$ & $\begin{array}{l}1684.24(1514.43- \\
1863.91)\end{array}$ & $41.09(36.98-45.44)$ & $\begin{array}{l}-0.57(-0.72 \text { to } \\
-0.41)\end{array}$ \\
\hline Female & $585.45(535.67-629.28)$ & ) $17.01(15.57-18.30)$ & $650.86(580.29-726.36)$ & $15.39(13.73-17.16)$ & $\begin{array}{l}-0.68(-0.94 \text { to } \\
-0.41)\end{array}$ \\
\hline High SDI & $150.17(145.09-155.05)$ & ) $11.67(11.30-12.04)$ & $159.39(146.90-173.32)$ & $10.82(9.95-11.78)$ & $\begin{array}{l}-0.56(-0.86 \text { to } \\
-0.26)\end{array}$ \\
\hline High-middle SDI & $519.63(484.58-562.70)$ & ) 32.08 (29.94-34.74) & $563.90(492.01-643.06)$ & $29.49(25.76-33.62)$ & $-0.27(-0.79$ to 0.26$)$ \\
\hline Middle SDI & $814.13(761.24-884.84)$ & ) 38.58 (36.17-41.73) & $\begin{array}{l}914.42(819.01- \\
1020.65)\end{array}$ & $34.02(30.52-37.87)$ & $\begin{array}{l}-1.21(-1.30 \text { to } \\
-1.12)\end{array}$ \\
\hline Low-middle SDI & $404.27(369.74-441.68)$ & $32.96(30.20-35.93)$ & $494.50(446.24-550.74)$ & $31.42(28.40-34.96)$ & $\begin{array}{l}-0.47(-0.63 \text { to } \\
-0.31)\end{array}$ \\
\hline Low SDI & $157.82(140.93-176.69)$ & ) $30.74(27.58-34.20)$ & 201.75 (177.99-227.16) & $28.97(25.53-32.56)$ & $\begin{array}{l}-0.52(-0.70 \text { to } \\
-0.35)\end{array}$ \\
\hline Andean Latin America & $2.21(1.97-2.44)$ & $4.93(4.38-5.47)$ & $2.55(2.03-3.16)$ & $4.30(3.42-5.33)$ & $\begin{array}{l}-1.52(-1.77 \text { to } \\
-1.26)\end{array}$ \\
\hline Australasia & $3.47(3.26-3.68)$ & $10.45(9.83-11.07)$ & $3.73(3.36-4.13)$ & $9.37(8.43-10.35)$ & $\begin{array}{l}-0.98(-1.23 \text { to } \\
-0.73)\end{array}$ \\
\hline Caribbean & $6.44(5.68-7.25)$ & $15.04(13.29-16.94)$ & $7.76(6.53-9.12)$ & $15.16(12.72-17.85)$ & $0.30(0.03-0.57)$ \\
\hline Central Asia & $9.57(9.18-9.99)$ & $13.15(12.64-13.71)$ & $12.21(10.78-13.87)$ & $13.61(12.03-15.39)$ & $0.44(-0.13$ to 1.01$)$ \\
\hline Central Europe & $24.58(23.82-25.38)$ & $15.10(14.66-15.58)$ & $21.53(18.62-24.72)$ & $12.83(11.11-14.77)$ & $\begin{array}{l}-1.64(-1.94 \text { to } \\
-1.33)\end{array}$ \\
\hline Central Latin America & $11.07(10.70-11.39)$ & $5.80(5.60-5.98)$ & $13.19(11.10-15.76)$ & $5.33(4.50-6.35)$ & $\begin{array}{l}-0.73(-1.13 \text { to } \\
-0.33)\end{array}$ \\
\hline $\begin{array}{l}\text { Central Sub-Saharan } \\
\text { Africa }\end{array}$ & $8.22(6.60-10.05)$ & $15.85(12.78-19.43)$ & $11.21(8.56-14.14)$ & $15.22(11.66-19.44)$ & $\begin{array}{l}-0.48(-0.58 \text { to } \\
-0.37)\end{array}$ \\
\hline East Asia & $902.48(831.26-988.48)$ & ) $53.20(49.08-58.20)$ & $\begin{array}{l}958.33(810.44- \\
1124.17)\end{array}$ & $46.29(39.30-54.04)$ & $\begin{array}{l}-1.40(-1.76 \text { to } \\
-1.04)\end{array}$ \\
\hline Eastern Europe & $30.88(30.17-31.68)$ & $10.84(10.60-11.11)$ & $30.89(27.09-35.03)$ & $10.68(9.38-12.08)$ & $0.39(-0.45$ to 1.23$)$ \\
\hline $\begin{array}{l}\text { Eastern Sub-Saharan } \\
\text { Africa }\end{array}$ & $67.97(53.54-79.34)$ & $40.48(31.60-47.32)$ & $90.08(67.60-110.87)$ & $38.43(28.90-46.98)$ & $\begin{array}{l}-0.50(-0.56 \text { to } \\
-0.44)\end{array}$ \\
\hline $\begin{array}{l}\text { High-income Asia } \\
\text { Pacific }\end{array}$ & $31.32(29.82-32.72)$ & $10.89(10.43-11.35)$ & $31.88(29.13-34.73)$ & $9.64(8.84-10.53)$ & $\begin{array}{l}-1.47(-1.69 \text { to } \\
-1.26)\end{array}$ \\
\hline $\begin{array}{l}\text { High-income North } \\
\text { America }\end{array}$ & $33.26(32.39-34.22)$ & $7.49(7.29-7.70)$ & $36.56(34.98-38.17)$ & $7.07(6.77-7.37)$ & $\begin{array}{l}-0.41(-0.62 \text { to } \\
-0.21)\end{array}$ \\
\hline $\begin{array}{l}\text { North Africa and Mid- } \\
\text { dle East }\end{array}$ & 85.93 (74.95-97.76) & $21.79(19.05-24.72)$ & $102.67(89.16-118.58)$ & 19.13 (16.69-21.98) & $\begin{array}{l}-1.26(-1.36 \text { to } \\
-1.15)\end{array}$ \\
\hline Oceania & $2.55(1.85-3.36)$ & $37.87(28.04-48.97)$ & $3.57(2.56-4.74)$ & 38.25 (27.89-49.97) & $-0.01(-0.17$ to 0.16$)$ \\
\hline South Asia & $404.50(368.60-445.60)$ & ) $32.24(29.45-35.47)$ & $508.46(449.58-584.00)$ & $31.11(27.48-35.71)$ & $\begin{array}{l}-0.40(-0.50 \text { to } \\
-0.29)\end{array}$ \\
\hline Southeast Asia & $299.55(273.30-327.78)$ & ) $55.30(50.66-60.38)$ & $364.17(316.98-418.04)$ & $52.27(45.66-59.98)$ & $\begin{array}{l}-0.49(-0.67 \text { to } \\
-0.32)\end{array}$ \\
\hline $\begin{array}{l}\text { Southern Latin } \\
\text { America }\end{array}$ & $3.99(3.77-4.22)$ & $6.17(5.83-6.52)$ & $4.27(3.91-4.66)$ & $5.54(5.08-6.05)$ & $\begin{array}{l}-1.05(-1.53 \text { to } \\
-0.57)\end{array}$ \\
\hline $\begin{array}{l}\text { Southern Sub-Saharan } \\
\text { Africa }\end{array}$ & $8.89(8.22-9.56)$ & $17.17(15.98-18.41)$ & $9.27(8.26-10.36)$ & $14.16(12.66-15.76)$ & $\begin{array}{l}-1.83(-2.03 \text { to } \\
-1.63)\end{array}$ \\
\hline Tropical Latin America & $17.55(16.98-18.15)$ & $8.77(8.48-9.06)$ & $18.58(17.42-19.70)$ & $7.47(7.00-7.92)$ & $\begin{array}{l}-1.76(-1.97 \text { to } \\
-1.55)\end{array}$ \\
\hline Western Europe & $57.43(55.22-59.59)$ & $9.50(9.16-9.85)$ & $56.03(52.22-59.85)$ & $8.36(7.80-8.93)$ & $\begin{array}{l}-1.27(-1.58 \text { to } \\
-0.96)\end{array}$ \\
\hline
\end{tabular}


Table 3 (continued)

\begin{tabular}{|c|c|c|c|c|}
\hline 2009 & & 2019 & & 2009-2019 \\
\hline A & Age-standardized & DALY & Age-s & EAPC \\
\hline No $* 10^{3}(95 \% \mathrm{CI})$ & $\begin{array}{l}\text { DALY rate/100,000 } \\
\text { No. }(95 \% \text { CI })\end{array}$ & No $* 10^{3}(95 \% \mathrm{CI})$ & $\begin{array}{l}\text { DALY rate/100,000 } \\
\text { No. }(95 \% \text { CI })\end{array}$ & No. $(95 \% \mathrm{CI})$ \\
\hline
\end{tabular}

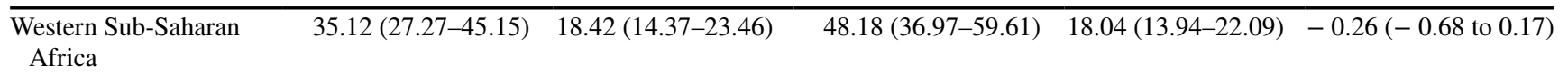

$D A L Y$ Disability-adjusted life year
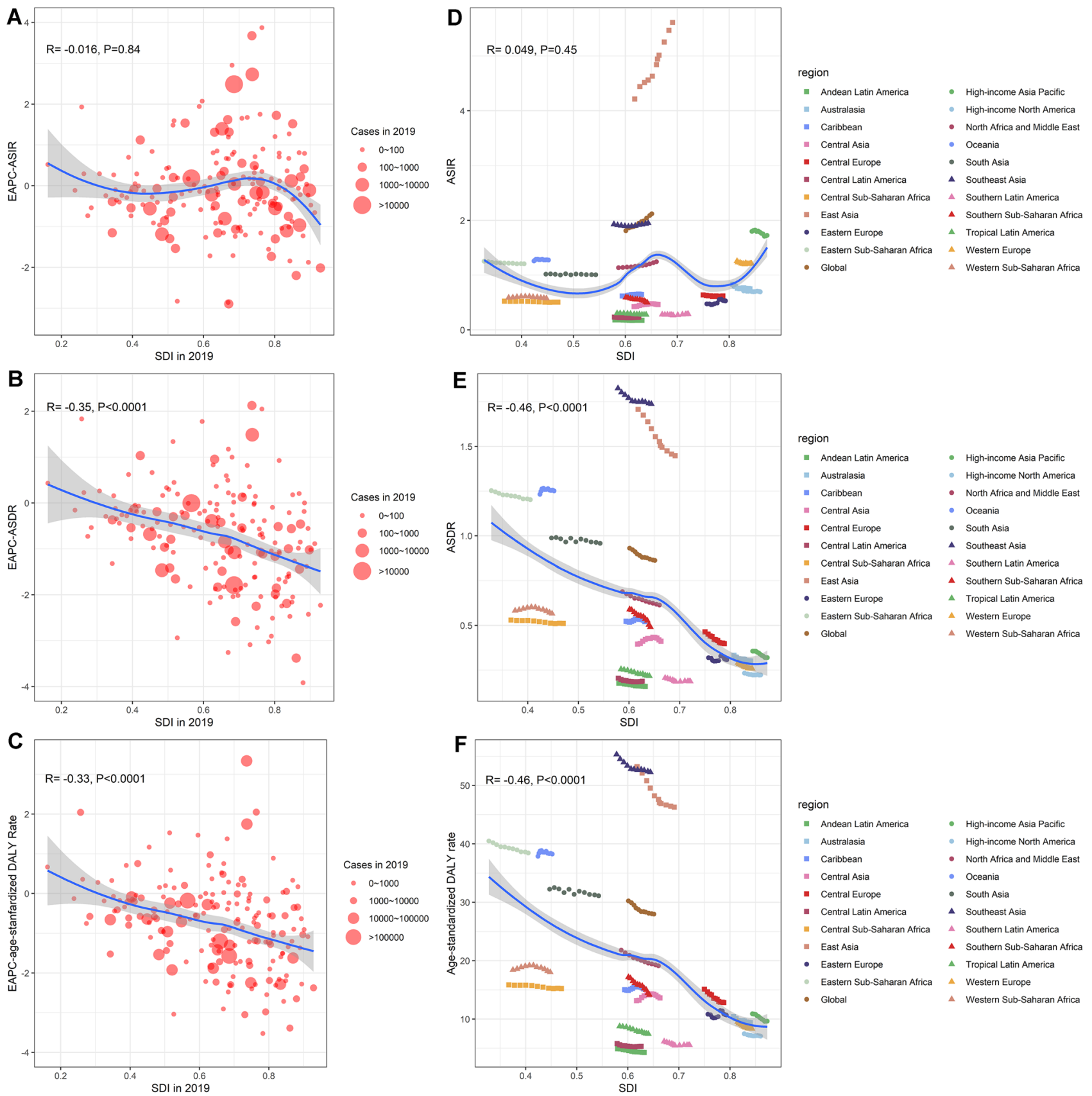

Fig. 4 Correlation between the global burden of NPC and SDI. A-C The correlation between EAPC of ASRs and SDI of 2019 in 204 countries or territories. D-F The change trends and correlation of ASRs and SDI from 2009 to 2019 in 21 regions 

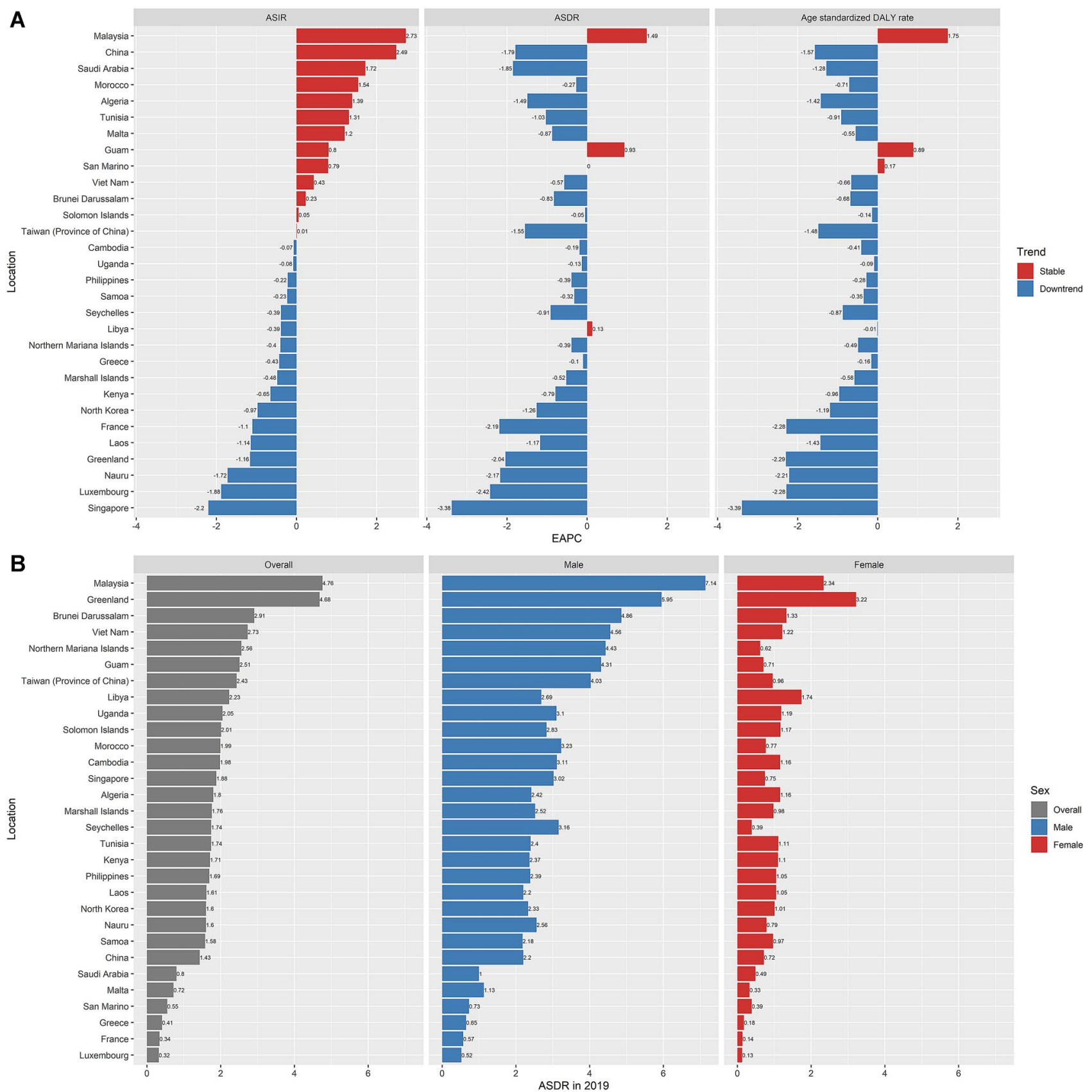

Fig. 5 NPC burden of high-incidence territories. A EAPCs of ASRs in high-incidence territories. B Gender-specific ASDR in high-incidence territories

that ASDR in high-incidence territories tended to be stable or decreased. Age-standardized DALY rate was the same as ASIR and ASDR (Tables S16-18). Malaysia had the highest ASDR in 2019 (ASDR =4.76), followed by Greenland (ASDR $=4.68)$. Among male patients, the highest ASDR was in Malaysia (ASDR $=7.14$ ), while among female patients in Greenland (ASDR =3.22). The ASDR of male patients was higher than that of female patients (Fig. 5B).

\section{The relationship between the incidence of NPC and age structure}

We divided the onset age of NPC into five age groups: under 10 years, 10-24 years, 25-49 years, 50-74 years and above 75 years, and analyzed the incidence and its rate in different age groups. In general, the number of patients with onset age greater than 50 years accounted for the highest proportion. This phenomenon was most evident in high SDI region 
(68.71\% in 2009 and $71.92 \%$ in 2019), and the proportion was yearly increasing. There was no significant difference in age structure between men and women. In addition, we found that the incidence cases of NPC in adolescents could not be ignored in low-middle and low SDI regions (Fig. S2A). Among all age groups, the highest incidence rate was found in patients over 50 years old, especially in the middleSDI area, while the gender difference was not significant (Fig. S2B).

\section{Dynamic distribution of risk factors associated with NPC-related mortality}

There are many main risk factors associated with NPC, and the GBD database shows three of them: smoking, occupational exposure to carcinogens (mainly formaldehyde), and alcohol use. We found that globally NPC-related death was most closely related to alcohol use from 2009 to 2019, especially in regions with relatively low SDI. The effect of exposure to carcinogens such as formaldehyde was relatively small in all regions and even weaker in regions with relatively high SDI (Figs. S3, S4).

\section{Discussion}

GBD 2019 updates and expands beyond GBD 2017 in ten ways, including update new data from 2018 to 2019, expand the number of countries, add new causes to the modeling framework, revise statistical methods, etc. Compared with the data from 1990 to 2017 in GBD2017, this study uses the data from 2009 to 2019 in GBD2019 to reduce the bias caused by long time span and better reflect the epidemiological trend of NPC in recent years [17]. In this study, we reported the incidence, mortality and DALY data of NPC based on GBD database, and analyzed the prevalence of NPC in the past 11 years by calculating EAPC values. In general, ASIR of NPC increased from 2009 to 2019 worldwide. Only the low SDI region showed a downward trend in ASIR. NPC in high-middle SDI region had the highest incidence cases, while the area with the most deaths was middle-SDI region, which warned about the heavy NPC burden in middle SDI areas. As reported in previous studies, the incidence rate of NPC had been declining in most areas of the world, especially in high-incidence territories such as Asia, Hong Kong and Taiwan [18-20]. The reason for this discrepancy might be that part of researches was relatively old and could not represent the general trend of the last decade. Data from GBD database are more effective, robust and comprehensive, which meets the requirement of accurately describing the epidemic trend of NPC. Another possible reason might be that advances in medical technology led to an increasing number of NPC patients being early detected. Compared with the old screening instruments, the sensitivity and specificity of EBV DNA in plasma samples in screening for NPC were $97.1 \%$ and $98.6 \%$, respectively [21]. Careful analysis was necessary while examining global trends in NPC incidence and comparing the results of this study with previous findings. More in-depth global statistics might be needed to verify the global burden of NPC further. In an era when the global NPC situation is still severe, the government and policymakers should raise the awareness of prevention and allocate medical resources rationally to reduce the incidence.

Compared with the increasing incidence rate, the decline in mortality of NPC in the world especially in high-incidence territories was encouraging. We believed this benefited from the advancement in medical professionalism, including enhanced understanding of the pathogenesis and risk factors, precise disease staging systems, individualized chemoradiotherapy (CRT) strategies, and the development of targeted therapy and immunotherapy. There was a significant negative correlation between mortality and SDI, indicating better prognosis of NPC in developed areas, possibly due to more advanced medical means. NPC was highly invasive and metastatic, but sensitive to both chemotherapy and radiotherapy (RT). RT is the mainstay treatment for non-metastatic NPC [1]. In recent years, RT technology has evolved from traditional two-dimensional RT to threedimensional conformal radiotherapy (3D-CRT), followed by more advanced intensity-modulated radiotherapy (IMRT) and stereotactic body radiotherapy (SBRT). More than 90\% of NPC patients achieved excellent local control after receiving high-quality IMRT [22]. For newly diagnosed nonmetastatic NPC patients, IMRT reduced the 5-year occurrence rate of locoregional failure to 7.4\% [23]. For locally advanced NPC, RT combined with chemotherapy (chemoradiotherapy, CRT) can achieve a better control rate than RT alone, allowing patients to harvest prolonged progressionfree survival and overall survival [24]. For recurrent or distant metastatic NPC, immunotherapy has gradually emerged as a promising treatment option that may elevate the treatment of NPC to a new level [25]. Other factors affecting mortality might be changes in lifestyle and reduced exposure to risk factors. The composition of NPC-related risk factors has undergone a series of changes along with the variations of the global environment in the last decade, which may have affected the epidemiological characteristics of NPC to a certain extent.

NPC-related risk factors include genetic factors, EBV infection, and environmental factors such as smoking, drinking, eating salted fish and exposure to carcinogens. EBV latently infects more than $90 \%$ of the world population and the majority of NPC are found to be positive for EBV infection [26]. EBV infection may be the most common causal agent of NPC besides genetic factors [1]. Unfortunately, 
the exact role of EBV in the development of NPC remains unclear [8]. The reason why EBV infection is ubiquitous but NPC is only prevalent in specific regions is still a mystery. Furthermore, considering that the genetic susceptibility to NPC is relatively stable in the population, it is reasonable to assume that the variation trend of NPC was mainly attributed to changes in environmental risk factors [18]. GBD database demonstrates that tobacco and alcohol use are two vital NPC-related risk factors. According to the World Health Organization (WHO), tobacco use in Asian countries such as China, Malaysia, India and Vietnam declined between 2000 and 2020. However, our data showed that ASIR values in these four countries increased gradually from 2009 to 2019. One possible reason is that the positive effects of tobacco control have been obscured by the growth of other risk factors. Further study found that alcohol consumption in many countries such as China, India and Vietnam increased between 2005 and 2016, which might offset the positive effects of smoking control on NPC prevention. What is puzzling is that ASR values of Malaysia rose continuously while its smoking rate was decreasing and its alcohol consumption has not increased in the past 10 years. Further investigations are needed to explain this phenomenon.

By analyzing the data, we found that the NPC mortality was significantly higher among men than women, indicating that female patients had a better prognosis, which was presumably associated with the disease stage at diagnosis, biological behaviors, sex hormones and their receptors [27-29]. A study showed that only $50.6 \%$ of female patients had locoregionally advanced NPC, much lower than the $71.9 \%$ of male patients [29]. Therefore, female patients might have an earlier disease onset, thereby improving the prognosis to some extent. According to previous studies, the number of male smokers and alcoholics was much higher than female [30, 31]. However, life behaviors and diagnostic delays did not fully explain the gender-specific differences in survival, and the intrinsic biological characteristics of the human body may play a more critical role. Maasberg et al. found in cloning experiments that androgens could triple the proliferation of tumor cells, while estrogens did not promote tumor cell growth [32]. Another study suggested that female sex was a significant protective prognostic factor for NPC regardless of tumor stage. However, this advantage persisted at premenopausal age, declined during menopause and disappeared at postmenopausal period [28]. Other possible explanations for sex-specific differences in prognosis include discrepancies in response to treatment and immune homeostasis $[33,34]$. Further studies are required to verify the genuine nature of these potential factors.

The incidence of NPC increase as population ages. It is undisputed that host genetics play an essential role, but the accumulation of mutations with age alone is inadequate to explain why the NPC incidence increases with age. One possible reason is that EBV infection often occurs at an early age, and the long-term stimulation of ubiquitous environmental factors interacts with genetic components, leading to impaired immune control of EBV over the years, thus developing into NPC many years later [5]. A significant proportion of adolescent NPC patients were discovered in the underdeveloped regions, leading to bimodality of the age-incidence curve at those areas, in which the earlier peak (under 25 years) might be associated with HLA gene or other NPC-related susceptibility genes [35].

This is the first study to systematically summarize the global epidemiological trends of NPC in the last decade. However, several deficiencies exist in this study. First, the reliability of the analysis depends on the accuracy and normalization of the data in GBD database. Unavoidably, there may be some undetected potential bias factors in GBD database when dealing with heterogeneous information from different databases. In some underdeveloped areas, obtained data often fail to fully reflect the authenticity of NPC burden because of the immaturity of the disease surveillance and death reporting system. Second, this study lacks sufficient data to determine trends of the disease by histological subtypes. Previous studies found that in the areas where NPC was prevalent, undifferentiated type accounted for the most and was always related to EBV infection, while the differentiated subtypes were more common in non-epidemic areas. Finally, EAPCs are used to evaluate the overall trends on a linear scale and cannot fully reflect the detailed trends of ASRs. To further reduce the global NPC burden, governments and health systems should vigorously advocate the primary prevention of the disease, including control the use of tobacco and alcohol, reduce the consumption of pickled food, increase the intake of fruits and vegetables and take protective measures when exposed to benzene and formaldehyde. In addition, other measures such as large-scale screening, efficient screening strategies, and advanced treatment modalities are also of paramount significance.

\section{Conclusions}

While the increase of nasopharyngeal carcinoma incidence was slight but progressive, mortality of NPC reduced considerably, possibly due to environmental and lifestyle modifications, intensive understanding of risk factors, population screening, precise disease staging systems, and advancement in medical criterion. Subgroup analysis by SDI demonstrated that high-middle and middle SDI regions had the highest burden of NPC. Morbidity and mortality of males significantly outnumbered that of females. The highest incidence occurs in people over 50 years of age, while in underdeveloped territories especially low-middle and low SDI regions, morbidity among adolescent patients could not be 
underestimated. The next decade should mainly focus on controllable etiological factors, including reducing tobacco and alcohol consumption, controlling the intake of pickled food, and avoiding contact with benzene and formaldehyde. In conclusion, medical policymakers should adjust their medical strategies according to the actual situation of the disease to further reduce the burden of NPC.

Supplementary Information The online version contains supplementary material available at https://doi.org/10.1007/s00405-021-06922-2.

Author contributions $\mathrm{HY}$ and $\mathrm{XY}$ contributed equally to the work. HY designed the study. HY, XY and MC wrote the main manuscript text. HY, YM and QT performed the figures and tables. SY reviewed the manuscript. All authors read and approved the final manuscript.

Funding This study was supported by Grants from Chinese Medicine Research Program of Zhejiang Province [2018ZZ014], Zhejiang Provincial Key Discipline of Traditional Chinese Medicine [2017XK-A32], the Fundamental Research Funds for the Central Universities [2021FZZX005-32], the Natural Science Foundation of Zhejiang Province of China [LSY19H160004].

Availability of data and materials The datasets generated during and/ or analyzed during the current study are available from the Global Health Data Exchange (GHDx) query tool (http://ghdx.healthdata.org/ gbd-results-tool).

\section{Declarations}

Conflict of interest The authors declare that the research was conducted in the absence of any commercial or financial relationships that could be construed as a potential conflict of interest.

Open Access This article is licensed under a Creative Commons Attribution 4.0 International License, which permits use, sharing, adaptation, distribution and reproduction in any medium or format, as long as you give appropriate credit to the original author(s) and the source, provide a link to the Creative Commons licence, and indicate if changes were made. The images or other third party material in this article are included in the article's Creative Commons licence, unless indicated otherwise in a credit line to the material. If material is not included in the article's Creative Commons licence and your intended use is not permitted by statutory regulation or exceeds the permitted use, you will need to obtain permission directly from the copyright holder. To view a copy of this licence, visit http://creativecommons.org/licenses/by/4.0/.

\section{References}

1. Chen YP, Chan A, Le QT, Blanchard P, Sun Y, Ma J (2019) Nasopharyngeal carcinoma. Lancet 394:64-80. https://doi.org/10.1016/ S0140-6736(19)30956-0

2. Tse LA, Yu IT, Mang OW, Wong SL (2006) Incidence rate trends of histological subtypes of nasopharyngeal carcinoma in Hong Kong. Br J Cancer 95:1269-1273. https://doi.org/10.1038/sj.bjc. 6603413

3. Marks JE, Phillips JL, Menck HR (1998) The National Cancer Data Base report on the relationship of race and national origin to the histology of nasopharyngeal carcinoma. Cancer-Am Cancer
Soc 83:582-588. https://doi.org/10.1002/(sici)1097-0142(19980 801)83:3\%3c582::aid-cncr29\%3e3.0.co;2-r

4. Bray F, Ferlay J, Soerjomataram I, Siegel RL, Torre LA, Jemal A (2018) Global cancer statistics 2018: GLOBOCAN estimates of incidence and mortality worldwide for 36 cancers in 185 countries. CA Cancer J Clin 68:394-424. https://doi.org/10.3322/caac. 21492

5. Chang ET, Adami HO (2006) The enigmatic epidemiology of nasopharyngeal carcinoma. Cancer Epidemiol Biomarkers Prev 15:1765-1777. https://doi.org/10.1158/1055-9965.EPI-06-0353

6. Li JH, Chia M, Shi W, Ngo D, Strathdee CA, Huang D et al (2002) Tumor-targeted gene therapy for nasopharyngeal carcinoma. Cancer Res 62:171-178

7. Tsao SW, Tsang CM, Lo KW (2017) Epstein-Barr virus infection and nasopharyngeal carcinoma. Philos Trans R Soc Lond B Biol Sci. https://doi.org/10.1098/rstb.2016.0270

8. Young LS, Yap LF, Murray PG (2016) Epstein-Barr virus: more than 50 years old and still providing surprises. Nat Rev Cancer 16:789-802. https://doi.org/10.1038/nrc.2016.92

9. Bei JX, Zuo XY, Liu WS, Guo YM, Zeng YX (2016) Genetic susceptibility to the endemic form of NPC. Chin Clin Oncol 5:15. https://doi.org/10.21037/cco.2016.03.11

10. Kanno M, Narita N, Fujimoto Y, Wakisaka N, Yoshizaki T, Kodaira T et al (2019) Third Epidemiological Analysis of Nasopharyngeal Carcinoma in the Central Region of Japan from 2006 to 2015. Cancers (Basel). https://doi.org/10.3390/cance rs 11081180

11. Du T, Chen K, Zheng S, Bao M, Huang Y, Wu K (2019) Association between alcohol consumption and risk of nasopharyngeal carcinoma: a comprehensive meta-analysis of epidemiological studies. Alcohol Clin Exp Res 43:2262-2273. https://doi.org/ 10.1111/acer.14184

12. Jia WH, Luo XY, Feng BJ, Ruan HL, Bei JX, Liu WS et al (2010) Traditional Cantonese diet and nasopharyngeal carcinoma risk: a large-scale case-control study in Guangdong, China. BMC Cancer 10:446. https://doi.org/10.1186/ 1471-2407-10-446

13. Yong SK, Ha TC, Yeo MC, Gaborieau V, McKay JD, Wee J (2017) Associations of lifestyle and diet with the risk of nasopharyngeal carcinoma in Singapore: a case-control study. Chin J Cancer 36:3. https://doi.org/10.1186/s40880-016-0174-3

14. Gallicchio L, Matanoski G, Tao XG, Chen L, Lam TK, Boyd K et al (2006) Adulthood consumption of preserved and nonpreserved vegetables and the risk of nasopharyngeal carcinoma: a systematic review. Int J Cancer 119:1125-1135. https://doi.org/ 10.1002/ijc. 21946

15. Olusanya BO, Ştefan S, Kim Y, Alcalde-Rabanal JE, Akinyemiju T, Koyanagi A et al (2020) Global burden of 369 diseases and injuries in 204 countries and territories, 1990-2019: a systematic analysis for the Global Burden of Disease Study 2019. Lancet 396:1204-1222. https://doi.org/10.1016/S0140-6736(20)30925-9

16. Fay MP, Tiwari RC, Feuer EJ, Zou Z (2006) Estimating average annual percent change for disease rates without assuming constant change. Biometrics 62:847-854. https://doi.org/10.1111/j.15410420.2006.00528.x

17. Hu CY, Wang WM, Chu XH, Ren ZH, Lyu J (2020) Global, regional, and national burden of nasopharyngeal carcinoma from 1990 to 2017—results from the Global Burden of Disease Study 2017. Head Neck 42:3243-3252. https://doi.org/10.1002/hed. 26378

18. Tang LL, Chen WQ, Xue WQ, He YQ, Zheng RS, Zeng YX et al (2016) Global trends in incidence and mortality of nasopharyngeal carcinoma. Cancer lett 374:22-30. https://doi.org/10.1016/j. canlet.2016.01.040

19. Zhang LF, Li YH, Xie SH, Ling W, Chen SH, Liu Q et al (2015) Incidence trend of nasopharyngeal carcinoma from 1987 to 2011 
in Sihui County, Guangdong Province, South China: an ageperiod-cohort analysis. Chin J Cancer 34:350-357. https://doi. org/10.1186/s40880-015-0018-6

20. Luo J, Chia KS, Chia SE, Reilly M, Tan CS, Ye W (2007) Secular trends of nasopharyngeal carcinoma incidence in Singapore, Hong Kong and Los Angeles Chinese populations, 1973-1997. Eur J Epidemiol 22:513-521. https://doi.org/10.1007/ s10654-007-9148-8

21. Chan K, Woo J, King A, Zee B, Lam W, Chan SL et al (2017) Analysis of plasma Epstein-Barr Virus DNA to screen for nasopharyngeal cancer. N Engl J Med 377:513-522. https://doi.org/ 10.1056/NEJMoa1701717

22. Kwong DL, Pow EH, Sham JS, McMillan AS, Leung LH, Leung WK et al (2004) Intensity-modulated radiotherapy for early-stage nasopharyngeal carcinoma: a prospective study on disease control and preservation of salivary function. Cancer-Am Cancer Soc 101:1584-1593. https://doi.org/10.1002/cncr.20552

23. Mao YP, Tang LL, Chen L, Sun Y, Qi ZY, Zhou GQ et al (2016) Prognostic factors and failure patterns in non-metastatic nasopharyngeal carcinoma after intensity-modulated radiotherapy. Chin J Cancer 35:103. https://doi.org/10.1186/s40880-016-0167-2

24. Nakanishi Y, Wakisaka N, Kondo S, Endo K, Sugimoto H, Hatano $M$ et al (2017) Progression of understanding for the role of Epstein-Barr virus and management of nasopharyngeal carcinoma. Cancer Metastasis Rev 36:435-447. https://doi.org/10. 1007/s10555-017-9693-x

25. Le QT, Colevas AD, O'Sullivan B, Lee A, Lee N, Ma B et al (2019) Current treatment landscape of nasopharyngeal carcinoma and potential trials evaluating the value of immunotherapy. J Natl Cancer Inst 111:655-663. https://doi.org/10.1093/jnci/djz044

26. Jha HC, Pei Y, Robertson ES (2016) Epstein-Barr virus: diseases linked to infection and transformation. Front Microbiol 7:1602. https://doi.org/10.3389/fmicb.2016.01602

27. Xiao G, Cao Y, Qiu X, Wang W, Wang Y (2013) Influence of gender and age on the survival of patients with nasopharyngeal carcinoma. BMC Cancer 13:226. https://doi.org/10.1186/ 1471-2407-13-226

28. OuYang PY, Zhang LN, Lan XW, Xie C, Zhang WW, Wang QX et al (2015) The significant survival advantage of female sex in nasopharyngeal carcinoma: a propensity-matched analysis. Br J Cancer 112:1554-1561. https://doi.org/10.1038/bjc.2015.70

29. Lu X, Wang FL, Guo X, Wang L, Zhang HB, Xia WX et al (2013) Favorable prognosis of female patients with nasopharyngeal carcinoma. Chin J Cancer 32:283-288. https://doi.org/10.5732/cjc. 012.10058

30. Islami F, Stoklosa M, Drope J, Jemal A (2015) Global and regional patterns of tobacco smoking and tobacco control policies. Eur Urol Focus 1:3-16. https://doi.org/10.1016/j.euf.2014. 10.001

31. Griswold MG, Fullman N, Hawley C, Arian N, Zimsen SR, Tymeson HD et al (2018) Alcohol use and burden for 195 countries and territories, 1990-2016: a systematic analysis for the Global Burden of Disease Study 2016. Lancet 392:1015-1035. https:// doi.org/10.1016/S0140-6736(18)31310-2

32. Maasberg M, Rotsch M, Jaques G, Enderle-Schmidt U, Weehle R, Havemann K (1989) Androgen receptors, androgen-dependent proliferation, and 5 alpha-reductase activity of small-cell lung cancer cell lines. Int J Cancer 43:685-691. https://doi.org/10. 1002/ijc. 2910430424

33. Schmetzer O, Florcken A (2012) Sex differences in the drug therapy for oncologic diseases. In: Handb Exp Pharmacol, pp 411-442. https://doi.org/10.1007/978-3-642-30726-3_19

34. Bouman A, Heineman MJ, Faas MM (2005) Sex hormones and the immune response in humans. Hum Reprod Update 11:411423. https://doi.org/10.1093/humupd/dmi008

35. Bray F, Haugen M, Moger TA, Tretli S, Aalen OO, Grotmol T (2008) Age-incidence curves of nasopharyngeal carcinoma worldwide: bimodality in low-risk populations and aetiologic implications. Cancer Epidemiol Biomarkers Prev 17:2356-2365. https:// doi.org/10.1158/1055-9965.EPI-08-0461

Publisher's Note Springer Nature remains neutral with regard to jurisdictional claims in published maps and institutional affiliations. 